Wright State University

From the SelectedWorks of Nikolai V. Priezjev

Fall October 19, 2020

\title{
Alternating shear orientation during cyclic loading facilitates yielding in amorphous materials
}

Nikolai Priezjev, Wright State University 


\title{
Alternating Shear Orientation During Cyclic Loading Facilitates Yielding in Amorphous Materials
}

\author{
Nikolai V. Priezjev (D)
}

\author{
(Submitted April 8, 2020; in revised form July 12, 2020; Accepted September 5, 2020; published online October 19, 2020)
}

\begin{abstract}
The influence of alternating shear orientation and strain amplitude of cyclic loading on yielding in amorphous solids is investigated using molecular dynamics simulations. The model glass is represented via a binary mixture that was rapidly cooled well below the glass transition temperature and then subjected to oscillatory shear deformation. It was shown that periodic loading at strain amplitudes above the critical value first induces structural relaxation via irreversible displacements of clusters of atoms during a number of transient cycles, followed by an increase in potential energy due to the formation of a system-spanning shear band. Upon approaching the critical strain amplitude from above, the number of transient cycles required to reach the yielding transition increases. Interestingly, it was found that when the shear orientation is periodically alternated in two or three dimensions, the number of transient cycles is reduced but the critical strain amplitude remains the same as in the case of periodic shear along a single plane. After the yielding transition, the material outside the shear band continues strain-induced relaxation, except when the shear orientation is alternated in three dimensions and the glass is deformed along the shear band with the imposed strain amplitude every third cycle.
\end{abstract}

Keywords metallic glasses, molecular dynamics simulations, oscillatory deformation, yielding transition

\section{Introduction}

Understanding the relationship between the local atomic structure of amorphous alloys and their mechanical and physical properties is important for various structural, biomedical and environmental applications (Ref 1-3). It is well accepted that in contrast to crystalline materials, where plastic deformation is governed by motion of topological line defects, or dislocations, the elementary plastic event in amorphous materials involves a collective rearrangement of small group of atoms, or a shear transformation (Ref 4, 5). The lack of crystalline order in metallic glasses results in relatively highyield strength, and, at the same time, if brought to a relaxed state, glasses can fail via sudden formation of shear bands where strain becomes localized along narrow layers $(\operatorname{Ref} 6,7)$. On the other hand, metallic glasses can be made more ductile if they are mechanically or thermally rejuvenated or, alternatively, formed by rapid cooling from the liquid state. In the past, a number of thermomechanical processing methods, such as cold rolling, high-pressure torsion, thermal cycling, elastostatic loading and irradiation, were developed to rejuvenate glasses and improve plasticity (Ref 8). Despite recent advances, however, the structural relaxation and critical behavior in metallic glasses during time-periodic mechanical deformation are yet to be fully understood.

Nikolai V. Priezjev, Department of Mechanical and Materials Engineering, Wright State University, Dayton, $\mathrm{OH} 45435$; and National Research University Higher School of Economics, Moscow, Russia 101000. Contact e-mail: nikolai.priezjev@wright.edu.
In recent years, considerable experimental efforts were devoted to examine fracture and fatigue properties of bulk metallic glasses and their composites (Ref 9). Generally, the fatigue life is determined by the number of loading cycles required to initiate and propagate a crack up to a critical size. Depending on the applied stress, chemical environment, temperature and cyclic frequency, the number of cycles to failure can vary over several orders of magnitude (Ref 10). In particular, the fatigue life is reduced with decreasing cyclic frequency due to longer time for the residual gas to adsorb on the sample surface. On the other hand, the number of cycles to failure can be increased by coating or surface finishing since cracks typically start at the free surface. However, the complete understanding of the fatigue crack initiation and propagation during cycling deformation of amorphous alloys and its dependence on the composition, loading conditions and thermal history is still missing.

In the last few years, the processes of structural relaxation, rejuvenation and yielding in amorphous materials under cyclic loading were extensively studied using atomistic simulations (Ref 11-36). In general, the results depend sensitively on the simulation model, preparation history, details of deformation protocol and system size. In the athermal limit, using quasistatic oscillatory shear deformation protocol, it was shown that following a number of periods, a disordered system reaches the so-called limit cycle where the trajectory of each atom becomes exactly reversible after one or more cycles, despite that during each subyield cycle large clusters of atoms can undergo cooperative displacements (Ref 13,16$)$. Interestingly, it was recently shown that highly stable glasses, which were produced either using the swap Monte Carlo algorithm (Ref 35) or via mechanical annealing (Ref 36), can be reversibly deformed over a relatively broad range of strain amplitudes. When the strain amplitude is above a critical value, the yielding transition usually occurs after a number of transient cycles and it is accompanied by the formation of a shear band across the system (Ref 20, 21, 24, 27, 28, 33). While during startup 
continuous shear deformation of well annealed glasses, the position of strain localization cannot be determined until the yielding strain (Ref 37), the location of a shear band during time periodic deformation can be identified at least several cycles before the yielding transition (Ref 33). Furthermore, it was previously demonstrated that mechanical annealing during small-amplitude cyclic shear can be accelerated by periodically alternating shear orientation in two or three spatial dimensions (Ref 30). It remains unclear, however, how the yielding transition and shear band formation are affected by cyclic deformation with alternating orientation of the shear plane near the critical strain amplitude.

In this paper, the effects of alternating shear orientation and strain amplitude on yielding and relaxation in binary glasses during cyclic loading are studied using molecular dynamics simulations. The binary mixture is initially rapidly cooled deep into the glass phase and then subjected to periodic shear applied either along a single plane or alternated in two or three dimensions. It will be shown that the number of transient cycles before yielding is reduced when an additional shear orientation is introduced in the deformation protocol at a given strain amplitude. Moreover, the critical strain amplitude, below which the glass undergoes relaxation toward lower potential energy states, remains the same for periodic shear along a single or alternating planes.

The rest of the paper is organized as follows. The details of the molecular dynamics simulation model and oscillatory shear deformation protocols are described in the next section. The time dependence of the potential energy and shear stress as well as the analysis of nonaffine displacements during cyclic loading are presented in section 3 . The results are briefly summarized in the last section.

\section{Molecular Dynamics Simulations}

The disordered solid is modeled via the binary LennardJones (LJ) mixture where the interaction between different types of atoms is strongly non-additive, thus preventing crystallization below the glass transition temperature (Ref 38). This model was first developed and its properties were thoroughly examined by Kob and Andersen (KA) using molecular dynamics simulations (Ref 38 ). The parametrization of the KA model is similar to the description of the amorphous metal-metalloid alloy $\mathrm{Ni}_{80} \mathrm{P}_{20}$ originally studied by Weber and Stillinger (Ref 39). In the KA model, any two atoms interact via the $\mathrm{LJ}$ potential given by

$$
V_{\alpha \beta}(r)=4 \varepsilon_{\alpha \beta}\left[\left(\frac{\sigma_{\alpha \beta}}{r}\right)^{12}-\left(\frac{\sigma_{\alpha \beta}}{r}\right)^{6}\right],
$$

with the following parameters: $\varepsilon_{A A}=1.0, \varepsilon_{A B}=1.5, \varepsilon_{B B}=0.5$, $\sigma_{A A}=1.0, \sigma_{A B}=0.8, \sigma_{B B}=0.88$ and $m_{A}=m_{B}$ (Ref 38). The system consists of 48000 of atoms of type $A$ and 12,000 $B$ type atoms, and the total number of atoms is fixed to 60,000 throughout the study. To speed up computations, the cutoff radius is set to $r_{c, \alpha \beta}=2.5 \sigma_{\alpha \beta}$. The numerical results are reported using the reduced $\mathrm{LJ}$ units of length, mass and energy, as follows: $\sigma=\sigma_{A A}, m=m_{A}$, and $\varepsilon=\varepsilon_{A A}$. The equations of motion were integrated in parallel using the velocity Verlet algorithm with the time step $\Delta t_{M D}=0.005 \tau$, where $\tau=\sigma \sqrt{m / \varepsilon}$ is the LJ time (Ref 40,41$)$. It should be commented that a more realistic interatomic potential might be required to predict the glass-forming ability of multicomponent amorphous alloys (Ref 42, 43).

We next briefly describe the preparation procedure and the deformation protocol. After equilibration at the temperature $T_{L J}=1.0 \varepsilon / k_{B}$ and density $\rho=\rho_{A}+\rho_{B}=1.2 \sigma^{-3}$, the binary mixture was rapidly cooled with the rate $10^{-2} \varepsilon / k_{B} \tau$ to the low temperature $T_{L J}=0.01 \varepsilon / k_{B}$ at constant volume. For reference, the critical temperature of the KA model at $\rho=1.2 \sigma^{-3}$ is $T_{c}=0.435 \varepsilon / k_{B}$, where $k_{B}$ is the Boltzmann constant (Ref 38). The typical scales in the KA model are $\varepsilon=120 k_{\mathrm{B}} \mathrm{K}, \sigma=$ $3.4 \AA$ and $\tau=0.3$ ps (Ref 38). In our setup, periodic boundary conditions were applied, and the temperature was regulated via the Nosé-Hoover thermostat (Ref 40, 41). The molecular dynamics simulations were carried out in the NVT ensemble. The linear size of the periodic box is $L=36.84 \sigma$.

After rapid cooling to the temperature $T_{L J}=0.01 \varepsilon / k_{\mathrm{B}}$, the binary glass was periodically sheared with the period $T=$ $5000 \tau$ at constant volume, as follows:

$\gamma(t)=\gamma_{0} \sin (2 \pi t / T)$,

where $\gamma_{0}$ is the strain amplitude in the vicinity of the yielding point, $0.065 \leqslant \gamma_{0} \leqslant 0.070$, and the oscillation frequency is $\omega=2 \pi / T=1.26 \times 10^{-3} \tau^{-1}$. The deformation was applied either along a single plane (the $x z$ plane), or alternated between two planes (the $x z$ and $y z$ planes), or alternated in all three directions (i.e., along the $x z, y z$ and $x y$ planes). The deformation protocols are the same as in the recent study on mechanical annealing under periodic shear (Ref 30$)$. In the present study, the data were acquired only for one realization of disorder due to the considerable computational demands. For example, a typical production run at a given strain amplitude during 2600 cycles required about 70 days using 40 processors.

\section{Results}

It is well realized that periodic deformation of rapidly quenched glasses can lead to progressively lower energy states if the temperature is sufficiently below the glass transition temperature and the strain amplitude is smaller than a critical value (Ref 12, 20, 23, 24, 44). The structural relaxation originates from irreversible rearrangements of groups of atoms, which can relocate to nearby minima in the potential energy landscape during strain (Ref 44). In the recent study, it was demonstrated that evolution to more relaxed states is accelerated when shear orientation is alternated in two or three spatial dimensions (Ref 30). During alternating loading, the potential energy landscape is periodically deformed in different directions and groups of atoms can rearrange to deeper energy minima. In the following analysis, the influence of the deformation protocol with alternating shear orientation on yielding and shear band formation is examined near the critical strain amplitude.

The time dependence of the potential energy minima during periodic shear along a single plane (the $x z$ plane) is presented in Fig. 1 for the strain amplitudes $0.065 \leqslant \gamma_{0} \leqslant 0.070$. Here, the data are reported at the end of each cycle, when the net strain is zero, and the time is expressed in terms of the oscillation period, $T=5000 \tau$. It can be observed that in all cases, the potential energy initially rapidly decreases from $U \approx-8.20 \varepsilon$, 
which corresponds to the state right after cooling with the rate $10^{-2} \varepsilon / k_{B} \tau$ to $T_{\mathrm{LJ}}=0.01 \varepsilon / k_{B}$, down to $U \approx-8.274 \varepsilon$ at $t=200 T$. The rapid decay of the potential energy is expected, since the glass is initially quenched at a relatively high cooling rate, and therefore, its local structure contains many highenergy clusters of atoms that are prone to rearrangement under external perturbation. Upon further cycling, the potential energy continues to decrease at a slower rate when the strain amplitude is below the critical value, i.e., $\gamma_{0} \leqslant 0.066$. These results are consistent with previous findings on mechanical annealing of periodically driven glasses at zero (Ref 12, 13, 20) and finite (Ref 23, 24, 30, 34) temperatures.

By contrast, periodic shear deformation at higher strain amplitudes, $0.067 \leqslant \gamma_{0} \leqslant 0.070$, leads to the yielding transition after a number of cycles, as shown in Fig. 1. It can be seen that at relatively large strain amplitudes, $\gamma_{0}=0.069$ and 0.070 , the abrupt increase in the potential energy occurs after about 300 and 200 cycles, respectively. Upon approaching the critical

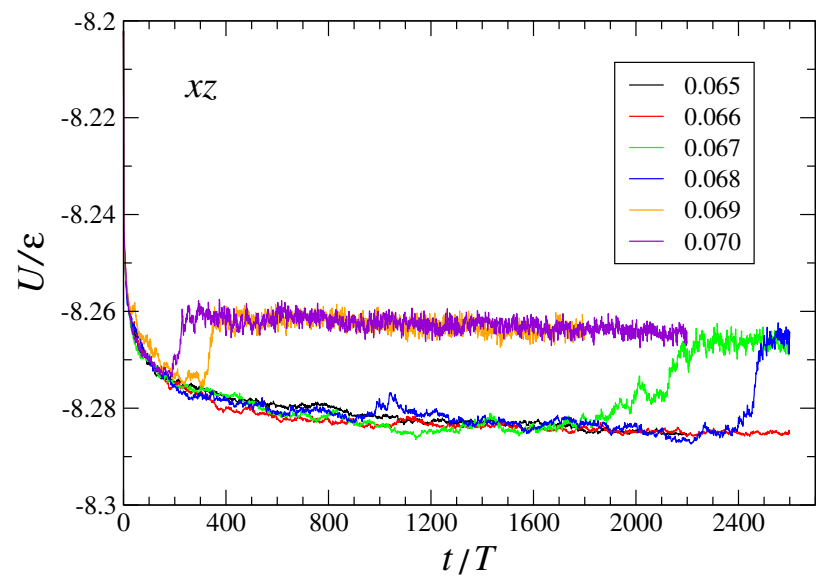

Fig. 1 (Color online) The potential energy minima as a function of the cycle number during periodic shear along the $x z$ plane. The strain amplitudes are indicated in the inset. The period of oscillation is $T=5000 \tau$ and the temperature is $T_{L J}=0.01 \varepsilon / k_{B}$

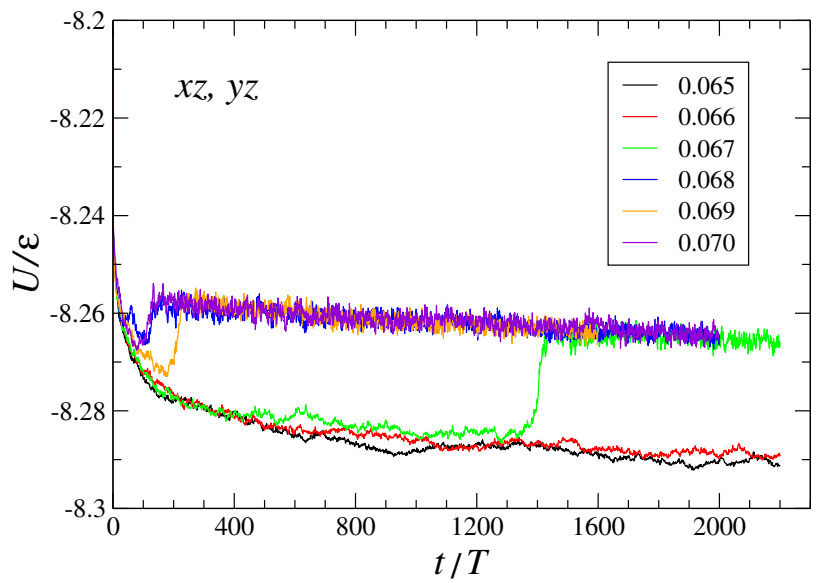

Fig. 2 (Color online) The variation of the potential energy minima vs. the cycle number during alternating shear along the $x z$ and $y z$ planes. The strain amplitudes are $\gamma_{0}=0.065$ (black), 0.066 (red), 0.067 (green), 0.068 (blue), 0.069 (orange) and 0.070 (velvet). The oscillation period is $T=5000 \tau$ value of the strain amplitude, $\gamma_{0}=0.067$, the number of transient cycles increases significantly. Somewhat unexpectedly, the yielding transition at the strain amplitude $\gamma_{0}=0.068$ is delayed by about 400 cycles with respect to the case $\gamma_{0}=0.067$, although the transient response is subject to large fluctuations (e.g., notice a local spike at $t \approx 1000 T$ in the blue curve in Fig. 1). It can be further observed that after the potential energy abruptly increases due to the formation of a shear band (to be discussed below), the region outside the shear band continues annealing, which is reflected in slightly negative slope of the potential energy as a function of the cycle number. This effect appears because strain is localized within the shear band, and the material outside the shear band is deformed at a strain amplitude smaller than the critical value. Similar results for the strain localization and relaxation of the solid phase as a function of accumulated strain were reported during cyclic athermal quasistatic deformation (Ref 28). Interestingly, the potential energy curves in Fig. 1 essentially

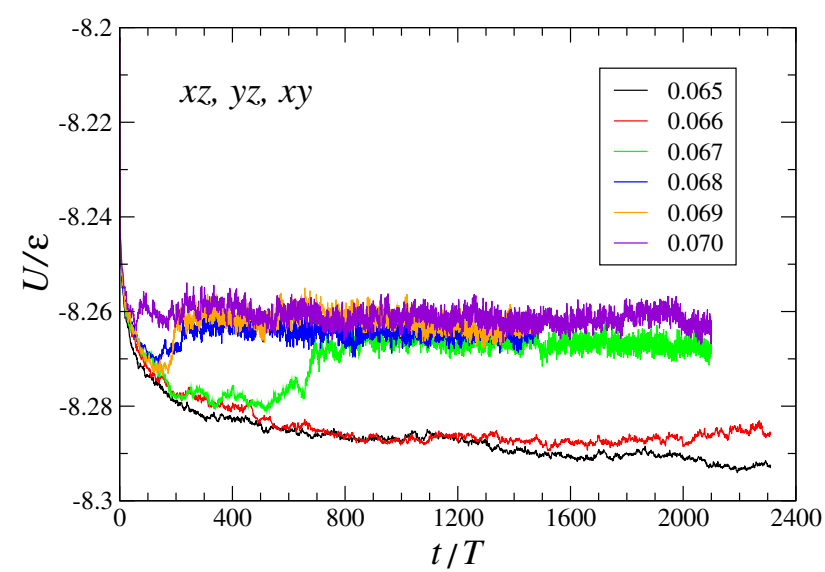

Fig. 3 (Color online) The potential energy at the end of each cycle during alternating shear along the $x z, y z$ and $x y$ planes. The values of the strain amplitude are listed in the legend. The time is measured in oscillation periods, $T=5000 \tau$

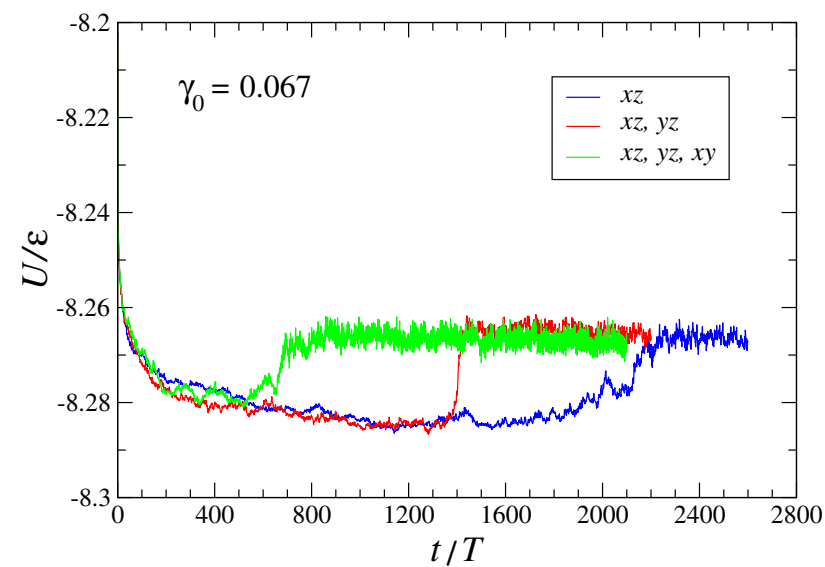

Fig. 4 (Color online) The potential energy minima as a function of time for the strain amplitude $\gamma_{0}=0.067$. The deformation protocols are: (1) periodic shear along the $x z$ plane, (2) alternating shear along the $x z$ and $y z$ planes, and (3) alternating shear along the $x z, y z$ and $x y$ planes. The same data for $\gamma_{0}=0.067$ as in Fig. 1, 2 and 3 
follow either one of the two energy levels (except during the yielding transition) for the strain amplitudes near the critical value $0.065 \leqslant \gamma_{0} \leqslant 0.070$

The results for the potential energy versus cycle number for the deformation protocol where the orientation of the shear

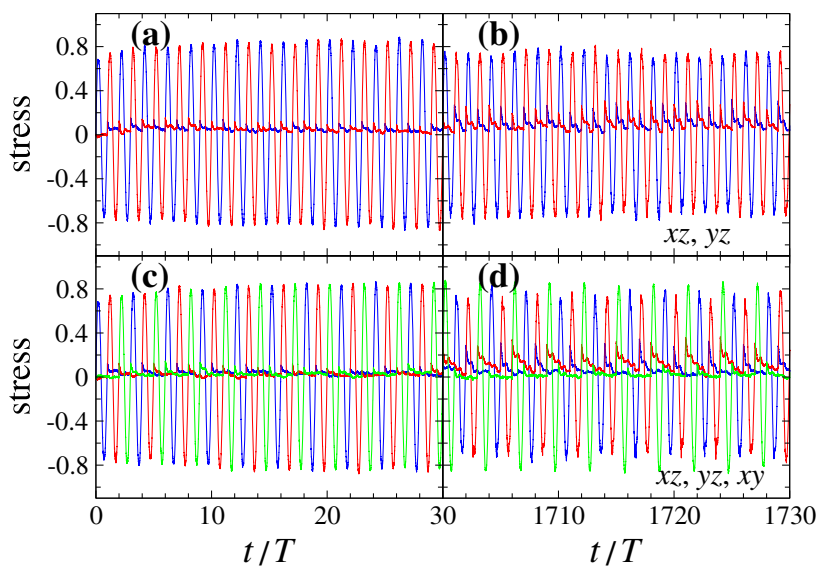

Fig. 5 (Color online) The time dependence of the shear stress (in units of $\varepsilon \sigma^{-3}$ ) for the strain amplitude $\gamma_{0}=0.067$. The upper panels show the data for alternating shear along the $x z$ and $y z$ planes, and the lower panels are for the alternating shear along the $x z, y z$ and $x y$ planes. The data for the planes of shear are denoted by the blue $(x z)$, red $(y z)$ and green $(x y)$ colors. The period is $T=5000 \tau$ plane is alternated between the $x z$ and $y z$ planes are shown in Fig. 2. The behavior is similar to the case of periodic shear along a single plane, except that the sharp yielding transition at the critical strain amplitude $\gamma_{0}=0.067$ occurs after about $1400 T$ rather than a gradual crossover after $2000 T$ at $\gamma_{0}=$ 0.067 reported in Fig. 1. Note also that the number of transient cycles decreases drastically for the strain amplitude $\gamma_{0}=0.068$, i.e., about 100 cycles for alternating loading in Fig. 2 and about 2500 cycles for periodic shear along a single plane in Fig. 1.

Furthermore, the data for the alternating shear along the $x z$, $y z$ and $x y$ planes are presented in Fig. 3 for the same strain amplitudes, $0.065 \leqslant \gamma_{0} \leqslant 0.070$. There are two main differences from the previous cases. First, the yielding transition at the critical strain amplitude $\gamma_{0}=0.067$ occurs much sooner than for the other deformation protocols, i.e., already after about 600 cycles. Second, after a number of transient cycles, a single shear band is formed along one of the planes for loading at the strain amplitudes $0.067 \leqslant \gamma_{0} \leqslant 0.070$. It means that one of the three shear orientations coincides with the plane parallel to the shear band, and therefore, the material outside the shear band is strained at the imposed $\gamma_{0}$ during one of the cycles, while the effective strain amplitude is reduced during the other two cycles. As a result, the annealing outside the shear band is suppressed (notice nearly constant energy levels after the transition), and the plateau levels for $t \gtrsim 800 T$ in Fig. 3 become progressively higher with increasing strain amplitude in the range $0.067 \leqslant \gamma_{0} \leqslant 0.070$.
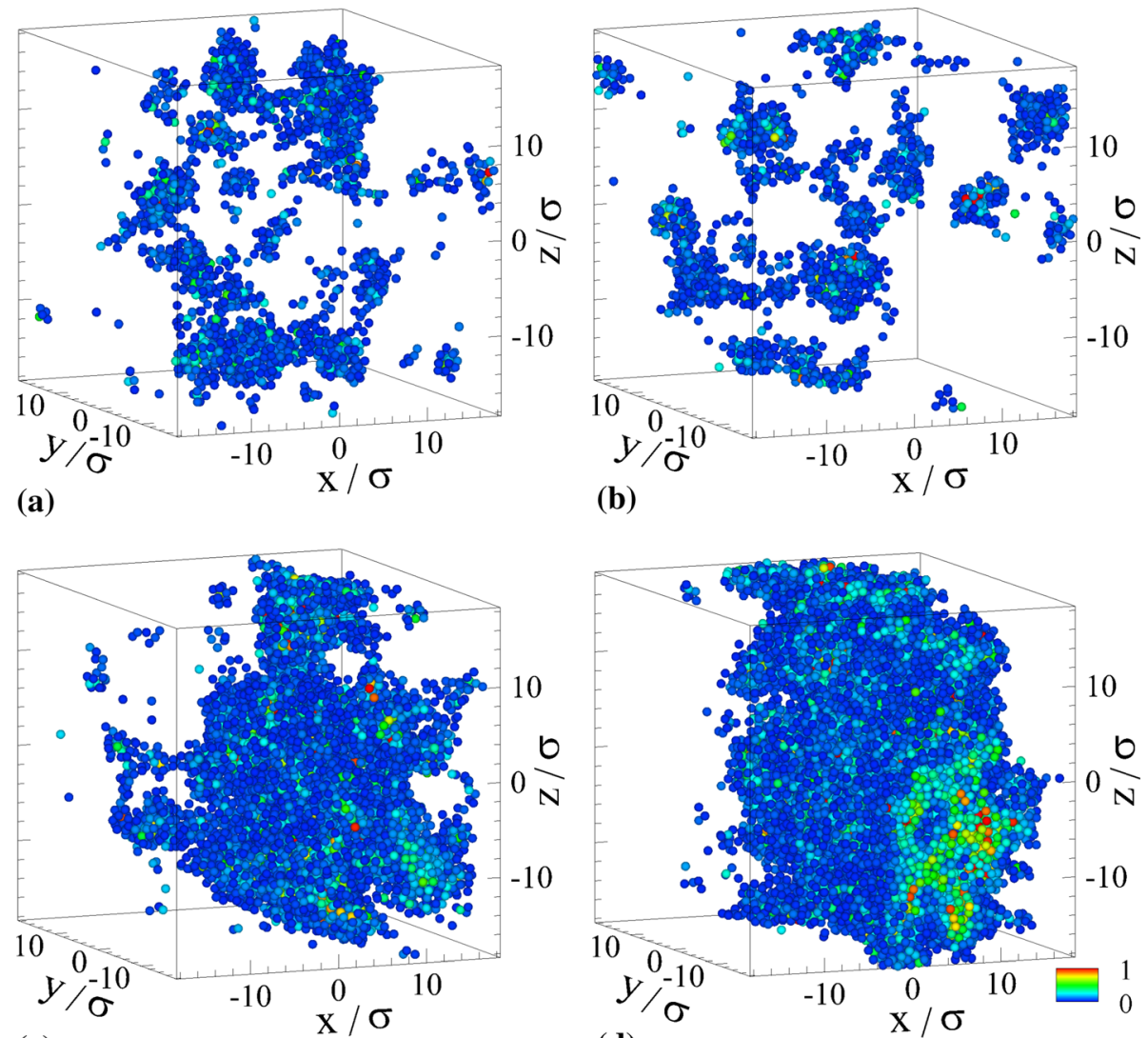

(c)

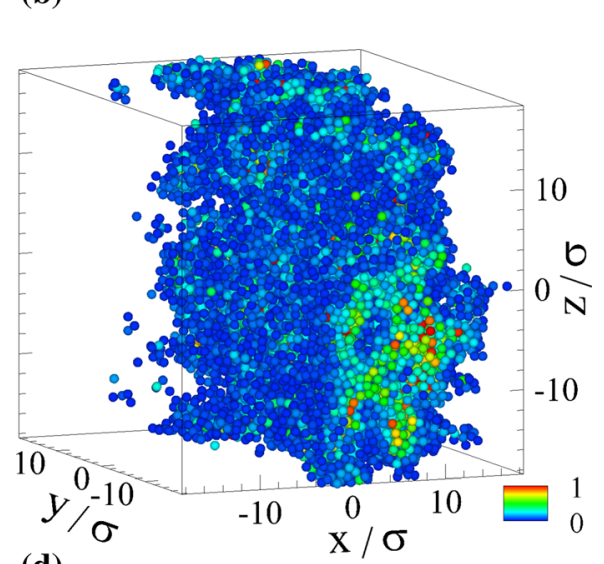

(d)

Fig. 6 (Color online) A sequence of snapshots during periodic shear along the $x z$ plane with the strain amplitude $\gamma_{0}=0.067$. The nonaffine measure is (a) $D^{2}(400 T, T)>0.04 \sigma^{2}$, (b) $D^{2}(1200 T, T)>0.04 \sigma^{2}$, (c) $D^{2}(2000 T, T)>0.04 \sigma^{2}$, and (d) $D^{2}(2400 T, T)>0.04 \sigma^{2}$. The colorcode for $D^{2}$ is specified in the legend. The oscillation period is $T=5000 \tau$. The atoms are not shown to scale 

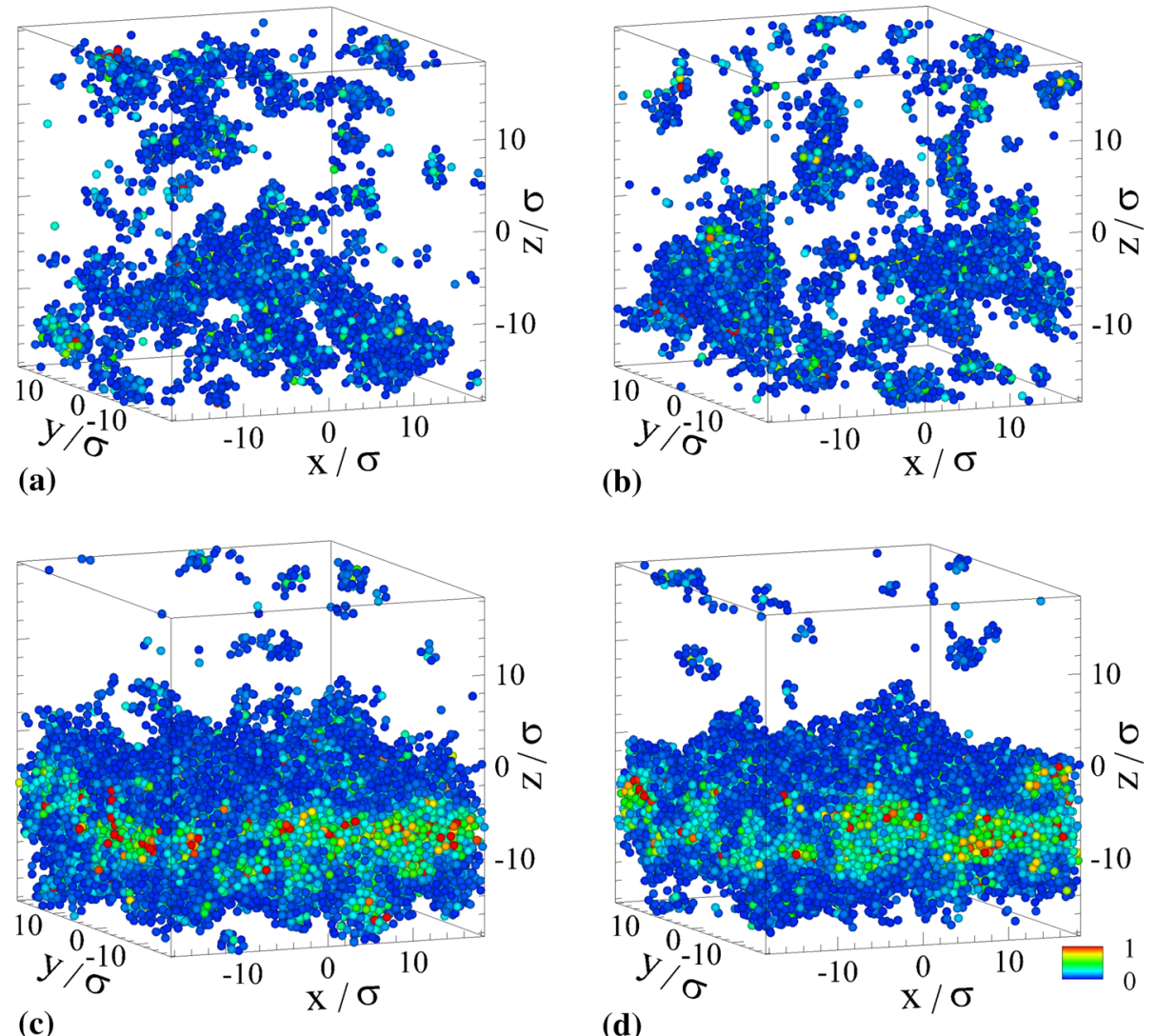

Fig. 7 (Color online) The snapshots of atomic configurations during alternating shear along the $x z$ and $y z$ planes with the strain amplitude $\gamma_{0}=0.067$. The nonaffine measure is (a) $D^{2}(400 T, T)>0.04 \sigma^{2}$, $D^{2}(2000 T, T)>0.04 \sigma^{2}$. The colorcode for $D^{2}$ is defined in the legend

(b) $D^{2}(1200 T, T)>0.04 \sigma^{2}$, (c) $D^{2}(1600 T, T)>0.04 \sigma^{2}$, and (d)

In order to facilitate comparison of the results for different deformation protocols, the potential energy curves are replotted in Fig. 4 for the critical strain amplitude $\gamma_{0}=0.067$. As is evident, the potential energies are roughly the same up to the yielding transition, and the number of shear cycles to reach the transition point is reduced for protocols where the shear orientation is periodically alternated in two and three dimensions. In the context of the previous study on mechanical annealing of periodically deformed glasses, where it was shown that relaxation is accelerated with each additional alternation of the shear orientation (Ref 30$)$, it is important to note that the critical value of the strain amplitude, $\gamma_{0}=0.067$, remains unchanged for different deformation protocols. We comment, however, that this conclusion is based on a limited number of cycles at the strain amplitude $\gamma_{0}=0.066$, as reported in Fig. 1, 2 and 3.

The variation of shear stress along the $x z, y z$ and $x y$ planes during alternating loading is shown in Fig. 5 for the strain amplitude $\gamma_{0}=0.067$. The data are presented during the first 30 cycles after thermal annealing and during 30 cycles after the yielding transition (see Fig. 4). It can be observed in Fig. 5(a) and (c) that for both deformation protocols, the stress amplitude initially increases, as the glass becomes more relaxed, and it saturates to a nearly constant level. These results are similar to the stress variation during periodic shear along a single plane of rapidly annealed binary glasses (Ref 23,24$)$. By contrast, the plastic flow within a shear band causes stress-strain hysteresis, and consequently, the shear stress at the end of each cycle remains finite, and it approaches zero during the following one or two cycles, as shown in Fig. 5(b) and (d). The exception from this behavior is the deformation along the shear band in the case of alternating shear in three dimensions; see the $x y$ stress component (the green curve) in Fig. 5(d). Note also that the stress amplitude during deformation along the $x y$ plane is significantly larger than the amplitudes along the other directions, which are determined by the maximum stress within the shear band.

It is interesting to note that although yielding mechanisms are different in amorphous and crystalline metals, the results of MD simulations have shown that dislocation nucleation governs the strength of nano-twinned polycrystals, which results in their softening below a critical twin thickness (Ref 45). More specifically, at sufficiently small values of a twinboundary spacing, twin-boundary migration originates from nucleation and motion of partial dislocations parallel to the twin planes (Ref 45).

The collective rearrangements of atoms during the relaxation stage as well as the formation of a shear band can be visualized by computing the so-called nonaffine displacements. We recall that the nonaffine displacement of an atom is defined with respect to its neighbors by using the matrix $\mathbf{J}_{i}$, which transforms the positions of neighboring atoms during the time interval $\Delta t$ and at the same time minimizes the following quantity (Ref 46): 


$$
\begin{gathered}
D^{2}(t, \Delta t)=\frac{1}{N_{i}} \sum_{j=1}^{N_{i}}\left\{\mathbf{r}_{j}(t+\Delta t)-\mathbf{r}_{i}(t+\Delta t)\right. \\
\left.-\mathbf{J}_{i}\left[\mathbf{r}_{j}(t)-\mathbf{r}_{i}(t)\right]\right\}^{2},
\end{gathered}
$$

where the summation is performed over atoms within a sphere of radius $1.5 \sigma$ and centered at $\mathbf{r}_{i}(t)$. It was previously found that the nonaffine measure is particularly well suited for identification of localized shear transformations in quiescent and deformed disordered solids (Ref 46-48). More recently, the analysis of nonaffine displacements was used to elucidate the structural relaxation dynamics and yielding during time periodic deformation (Ref 17, 19, 21, 23-25, 30, 33, 34) and thermal processing (Ref 49-53) of binary glasses.

The sequences of snapshots of atomic configurations for different deformation protocols at the critical strain amplitude $\gamma_{0}=0.067$ are displayed in Fig. 6, 7 and 8. Here, the nonaffine measure was computed for two consecutive configurations at zero strain, and the time interval in Eq 3 is set to $\Delta t=T$. For visualization of irreversible rearrangements during one shear cycle, only atoms with relatively large nonaffine displacements are shown, i.e., $D^{2}(n T, T)>0.04 \sigma^{2}$, where $n$ is the integer. The typical cage size at the density $\rho=1.2 \sigma^{-3}$ is $r_{c} \approx 0.1 \sigma$ (Ref 11). As shown in Fig. 6(a) and (b), the structural relaxation during mechanical annealing at $\gamma_{0}=0.067$ (the blue curve, $t \lesssim 1400 T$ in Fig. 4) proceeds via rearrangement of relatively small clusters of atoms. Interestingly, the shear band in Fig. 6(c) is only partially formed along the $y z$ plane as the yielding transition occurs gradually during several hundred periods (i.e., $1600 T \lesssim t \lesssim 2200 T$ in Fig. 4). Finally, the snapshot at Fig. 6(d) confirms that the shear band is fully developed after yielding, whereas irreversible displacements outside the shear band are nearly absent since the effective strain amplitude in that region is smaller than $\gamma_{0}=0.067$.

The structural relaxation before and after the yielding transition at $\gamma_{0}=0.067$ is similar in the case of alternating shear along the $x z$ and $y z$ panes, as evident from Fig. 7. Notice, however, that the shear band is formed at $t=1600 T$, since the sharp yielding transition occurs already at $t \approx 1400 T$ (see Fig. 4). While the orientation of the shear band in Fig. 6 and 8 is difficult to predict, the deformation protocol with alternating shear along the $x z$ and $y z$ planes imposes formation of the shear band along the perpendicular plane (i.e., along the $x y$ plane). Further, the appearance of a nearly percolating cluster of mobile atoms in Fig. 8(a) precedes the yielding transition when shear orientation is alternated in all three dimensions. Moreover, once the shear band is formed along the $x y$ plane, as illustrated in Fig. 8(b)-(d), every third cycle with $\gamma_{0}=0.067$ along the $x y$ plane induces relatively large irreversible rearrangements outside the shear band. It should also be commented that only one shear band is formed at the largest strain amplitude $\gamma_{0}=$ 0.070 for alternating shear along three dimensions (not shown). Taken together, the evolution of spatial distributions of atoms with large nonaffine displacements during cyclic loading at the
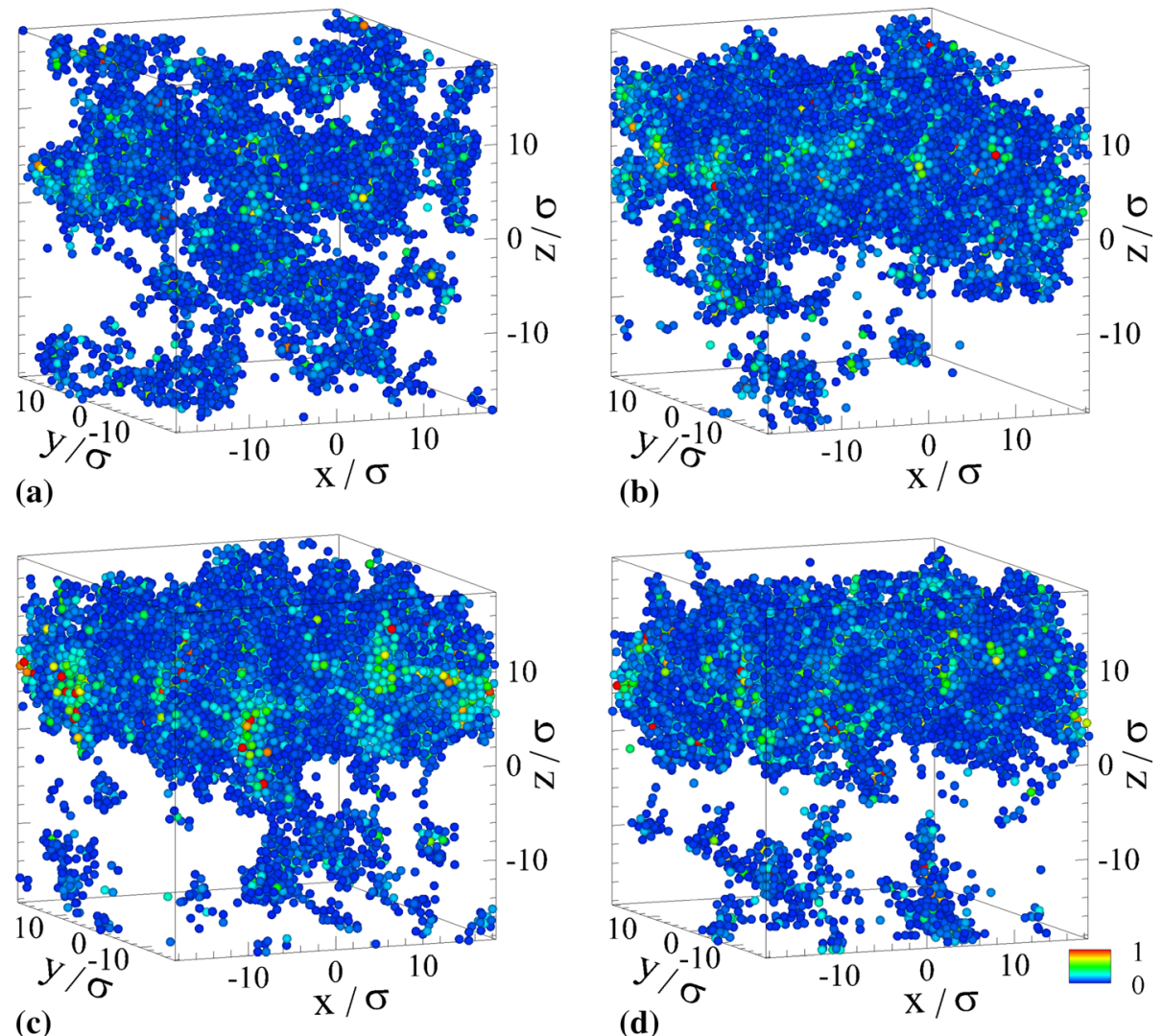

Fig. 8 (Color online) The snapshots of the system during alternating shear along the $x z, y z$ and $x y$ planes with the strain amplitude $\gamma_{0}=0.067$. The nonaffine measure is (a) $D^{2}(400 T, T)>0.04 \sigma^{2}$, (b) $D^{2}(800 T, T)>0.04 \sigma^{2}$, (c) $D^{2}(1200 T, T)>0.04 \sigma^{2}$, and (d) $D^{2}(2000 T, T)>0.04 \sigma^{2}$. The colorcode for $D^{2}$ is shown in the legend 
critical strain amplitude, shown in Fig. 6, 7 and 8, correlates well with the onset of yielding reported in Fig. 4.

We finally comment that the formation of shear bands and subsequent fatigue cracking was observed experimentally in metallic glasses under cyclic loading (Ref 54, 55). Moreover, upon reducing sample size down to the micron scale, both the maximum stress and the number of cycles to failure increase significantly during cyclic compression or bending tests (Ref 56). Although the system size in the present study is only about $10 \mathrm{~nm}$ and the surface effects for the shear band initiation are not included due to periodic boundary conditions, the results of MD simulations indicate that the number of cycles to form a shear band is reduced for loading along alternating directions, while the critical strain amplitude remains unaffected by the deformation protocol. In the future, it will be instructive to verify these predictions using fatigue experiments.

\section{Conclusions}

In summary, molecular dynamics simulations were carried out to examine the influence of alternating shear orientation during periodic shear of amorphous materials near the critical strain amplitude. We considered a binary glass rapidly quenched well below the glass transition temperature and then periodically deformed along a single plane or alternating planes in two or three spatial dimensions. It was found that at strain amplitudes below the critical value, the glass continues exploring lower potential energy states via collective, irreversible rearrangements of atoms. By contrast, at the critical strain amplitude and above, the structural relaxation is followed by the yielding transition and formation of a shear band along one of the planes. It was shown that the number of transient cycles before yielding is reduced when an additional alternation of shear orientation is included in the deformation protocol at a given strain amplitude.

Interestingly, following the yielding transition, the glass outside the shear band continues to relax, since the effective strain amplitude in the solid domain is reduced when shear is applied along a single plane or alternated between two planes. In the case of alternating shear orientation in all three dimensions, however, cyclic shear during one of the three periods is applied along the shear band, and the rest of the material is deformed with the imposed strain amplitude, leading to a nearly constant level of the potential energy. Furthermore, the deformation protocol with alternating shear along two planes imposes the formation of the shear band along the perpendicular plane, while for other protocols the orientation of the shear band cannot be easily predicted. These results are important for the development of processing methods that require precise control of relaxation and yielding in amorphous solids.

\section{Acknowledgments}

Financial support from the National Science Foundation (CNS1531923) is gratefully acknowledged. The article was prepared within the framework of the HSE University Basic Research Program and funded in part by the Russian Academic Excellence Project '5-100'. The numerical simulations were carried out at Wright State University's Computing Facility and the Ohio
Supercomputer Center. The molecular dynamics simulations were performed using the LAMMPS code developed at Sandia National Laboratories (Ref 40).

\section{Conflict of interest}

The author declares that he has no conflict of interest.

\section{References}

1. J.C. Qiao, Q. Wang, J.M. Pelletier, H. Kato, R. Casalini, D. Crespo, E. Pineda, Y. Yao, and Y. Yang, Structural Heterogeneities and Mechanical Behavior of Amorphous Alloys, Prog. Mater. Sci., 2019, 104, p 250

2. H.F. Li and Y.F. Zheng, Recent Advances in Bulk Metallic Glasses for Biomedical Applications, Acta Biomater, 2016, 36, p 1

3. L.-C. Zhang, Z. Jia, F. Lyu, S.-X. Liang, and J. Lu, A Review of Catalytic Performance of Metallic Glasses in Wastewater Treatment: Recent Progress and Prospects, Prog. Mater. Sci., 2019, 105, p 100576

4. F. Spaepen, A Microscopic Mechanism for Steady State Inhomogeneous Flow in Metallic Glasses, Acta Metall., 1977, 25, p 407

5. A.S. Argon, Plastic Deformation in Metallic Glasses, Acta Metall, 1979, 27, p 47

6. Y.Q. Cheng and E. Ma, Atomic-Level Structure and Structure-Property Relationship in Metallic Glasses, Prog. Mater. Sci., 2011, 56, p 379

7. R. Hubek, S. Hilke, F.A. Davani, M. Golkia, G.P. Shrivastav, S.V. Divinski, H. Rosner, J. Horbach, and G. Wilde, Shear Bands in Monolithic Metallic Glasses: Experiment, Theory, and Modeling, Front. Mater, 2020, 7, p 144

8. Y. Sun, A. Concustell, and A.L. Greer, Thermomechanical Processing of Metallic Glasses: Extending the Range of the Glassy State, Nat. Rev. Mater., 2016, 1, p 16039

9. B.A. Sun and W.H. Wang, The Fracture of Bulk Metallic Glasses, Prog. Mater. Sci., 2015, 74, p 211

10. H. Jia, G. Wang, S. Chen, Y. Gao, W. Li, and P.K. Liaw, Fatigue and Fracture Behavior of Bulk Metallic Glasses and Their Composites, Prog. Mater. Sci., 2018, 98, p 168

11. N.V. Priezjev, Heterogeneous Relaxation Dynamics in Amorphous Materials Under Cyclic Loading, Phys. Rev. E, 2013, 87, p 052302

12. D. Fiocco, G. Foffi, and S. Sastry, Oscillatory Athermal Quasistatic Deformation of a Model Glass, Phys. Rev. E, 2013, 88, p 020301(R)

13. I. Regev, T. Lookman, and C. Reichhardt, Onset of Irreversibility and Chaos in Amorphous Solids Under Periodic Shear, Phys. Rev. E, 2013, 88, p 062401

14. N.V. Priezjev, Dynamical Heterogeneity in Periodically Deformed Polymer Glasses, Phys. Rev. E, 2014, 89, p 012601

15. N.V. Priezjev, The Effect of a Reversible Shear Transformation on Plastic Deformation of an Amorphous Solid, J. Phys. Condens. Matter, 2015, 27, p 435002

16. I. Regev, J. Weber, C. Reichhardt, K.A. Dahmen, and T. Lookman, Reversibility and Criticality in Amorphous Solids, Nat. Commun., 2015, 6, p 8805

17. N.V. Priezjev, Reversible Plastic Events During Oscillatory Deformation of Amorphous Solids, Phys. Rev. E, 2016, 93, p 013001

18. T. Kawasaki and L. Berthier, Macroscopic Yielding in Jammed Solids is Accompanied by a Non-Equilibrium First-Order Transition in Particle Trajectories, Phys. Rev. E, 2016, 94, p 022615

19. N.V. Priezjev, Nonaffine Rearrangements of Atoms in Deformed and Quiescent Binary Glasses, Phys. Rev. E, 2016, 94, p 023004

20. P. Leishangthem, A.D.S. Parmar, and S. Sastry, The Yielding Transition in Amorphous Solids Under Oscillatory Shear Deformation, Nat. Commun., 2017, 8, p 14653

21. N.V. Priezjev, Collective Nonaffine Displacements in Amorphous Materials During Large-Amplitude Oscillatory Shear, Phys. Rev. E, 2017, 95, p 023002

22. M. Fan, M. Wang, K. Zhang, Y. Liu, J. Schroers, M.D. Shattuck, and C.S. O'Hern, The Effects of Cooling Rate on Particle Rearrangement Statistics: Rapidly Cooled Glasses are More Ductile and Less Reversible, Phys. Rev. E, 2017, 95, p 022611 
23. N.V. Priezjev, Molecular Dynamics Simulations of the Mechanical Annealing Process in Metallic Glasses: Effects of Strain Amplitude and Temperature, J. Non-Cryst. Solids, 2018, 479, p 42

24. N.V. Priezjev, The Yielding Transition in Periodically Sheared Binary Glasses at Finite Temperature, Comput. Mater. Sci., 2018, 150, p 162

25. N.V. Priezjev, Slow Relaxation Dynamics in Binary Glasses During Stress-Controlled, Tension-Compression Cyclic Loading, Comput. Mater. Sci., 2018, 153, p 235

26. N.V. Priezjev and M.A. Makeev, The Influence of Periodic Shear on Structural Relaxation and Pore Redistribution in Binary Glasses, $J$. Non-Cryst. Solids, 2019, 506, p 14

27. N.V. Priezjev and M.A. Makeev, Structural Transformations During Periodic Deformation of Low-Porosity Amorphous Materials, Model. Simul. Mater. Sci. Eng., 2019, 27, p 025004

28. A.D.S. Parmar, S. Kumar, and S. Sastry, Strain Localization Above the Yielding Point in Cyclically Deformed Glasses, Phys. Rev. X, 2019, 9, p 021018

29. Z.-Y. Zhou, H.-L. Peng, and H.-B. Yu, Structural Origin for VibrationInduced Accelerated Aging and Rejuvenation in Metallic Glasses, $J$ Chem. Phys., 2019, 150, p 204507

30. N.V. Priezjev, Accelerated Relaxation in Disordered Solids Under Cyclic Loading with Alternating Shear Orientation, J. Non-Cryst. Solids, 2019, 525, p 119683

31. G.-J. Lyu, J.-C. Qiao, Y. Yao, J.-M. Pelletier, D. Rodney, J. Morthomas, and C. Fusco, Dynamic Correspondence Principle in the Viscoelasticity of Metallic Glasses, Scr. Mater., 2020, 174, p 39

32. E. Schinasi-Lemberg and I. Regev, Annealing and Rejuvenation in a Two-Dimensional Model Amorphous Solid Under Oscillatory Shear, Phys. Rev. E, 2020, 101, p 012603

33. N.V. Priezjev, Shear Band Formation in Amorphous Materials Under Oscillatory Shear Deformation, Metals, 2020, 10, p 300

34. P.K. Jana and N.V. Priezjev, Structural Relaxation in Amorphous Materials Under Cyclic Tension-Compression Loading, J. Non-Cryst. Solids, 2020, 540, p 120098

35. W.-T. Yeh, M. Ozawa, K. Miyazaki, T. Kawasaki, and L. Berthier, Glass Stability Changes the Nature of Yielding Under Oscillatory Shear, Phys. Rev. Lett., 2020, 124, p 225502

36. H. Bhaumik, G. Foffi, S. Sastry, The Role of Annealing in Determining the Yielding Behavior of Glasses Under Cyclic Shear Deformation, a rXiv:1911.12957

37. N.V. Priezjev, Spatiotemporal Analysis of Nonaffine Displacements in Disordered Solids Sheared Across the Yielding Point, Metall. Mater. Trans. A, 2020, 51, p 3713

38. W. Kob and H.C. Andersen, Testing Mode-Coupling Theory for a Supercooled Binary Lennard-Jones Mixture: The van Hove Correlation Function, Phys. Rev. E, 1995, 51, p 4626

39. T.A. Weber and F.H. Stillinger, Local Order and Structural Transitions in Amorphous Metal-Metalloid Alloys, Phys. Rev. B, 1985, 31, p 1954

40. S.J. Plimpton, Fast Parallel Algorithms for Short-Range Molecular Dynamics, J. Comp. Phys., 1995, 117, p 1
41. M.P. Allen and D.J. Tildesley, Computer Simulation of Liquids, Clarendon, Oxford, 1987

42. M.H. Yang, J.H. Li, B.X. Liu, and J.B. Liu, Atomistic Simulations to Predict Favored Glass-Formation Composition and Ion-Beam-Mixing of Nano-Multiple-Metal-Layers to Produce Ternary Amorphous Films, Metals, 2018, 8, p 129

43. F. Chen, S. Cheng, J. Liu, J. Li, S. Zhao, S. Li, Q. Yang, and B. Liu, Atomistic Design Favored Compositions and Atomic-Level Structure of Mg-Ca-Ag Ternary Metallic Glasses, AIP Advances, 2019, 9, p 075025

44. D.J. Lacks and M.J. Osborne, Energy Landscape Picture of Overaging and Rejuvenation in a Sheared Glass, Phys. Rev. Lett., 2004, 93, p 255501

45. X. Li, Y. Wei, L. Lu, K. Lu, and H. Gao, Dislocation Nucleation Governed Softening and Maximum Strength in Nano-Twinned Metals, Nature, 2010, 464, p 877

46. M.L. Falk and J.S. Langer, Dynamics of Viscoplastic Deformation in Amorphous Solids, Phys. Rev. E, 1998, 57, p 7192

47. B. Utter and R.P. Behringer, Experimental Measures of Affine and Nonaffine Deformation in Granular Shear, Phys. Rev. Lett., 2008, 100, p 208302

48. H.L. Peng, M.Z. Li, and W.H. Wang, Structural Signature of Plastic Deformation in Metallic Glasses, Phys. Rev. Lett., 2011, 106, p 135503

49. N.V. Priezjev, Atomistic Modeling of Heat Treatment Processes for Tuning the Mechanical Properties of Disordered Solids, J. Non-Cryst. Solids, 2019, 518, p 128

50. N.V. Priezjev, The Effect of Cryogenic Thermal Cycling on Aging, Rejuvenation, and Mechanical Properties of Metallic Glasses, J. NonCryst. Solids, 2019, 503, p 131

51. Q.-L. Liu and N.V. Priezjev, The Influence of Complex Thermal Treatment on Mechanical Properties of Amorphous Materials, Comput. Mater. Sci., 2019, 161, p 93

52. N.V. Priezjev, Potential Energy States and Mechanical Properties of Thermally Cycled Binary Glasses, J. Mater. Res., 2019, 34, p 2664

53. N.V. Priezjev, Aging and Rejuvenation During Elastostatic Loading of Amorphous Alloys: A Molecular Dynamics Simulation Study, Comput. Mater. Sci., 2019, 168, p 125

54. M. Freels, G.Y. Wang, W. Zhang, P.K. Liaw, and A. Inoue, Cyclic Compression Behavior of a Cu-Zr-Al-Ag Bulk Metallic Glass, Intermetallics, 2011, 19, p 1174

55. X.D. Wang, R.T. Qu, Z.Q. Liu, and Z.F. Zhang, Shear Band-Mediated Fatigue Cracking Mechanism of Metallic Glass at High Stress Level, Mater. Sci. Eng. A, 2015, 627, p 336

56. D. Jang, R. Maass, G. Wang, P.K. Liaw, and J.R. Greer, Fatigue Deformation of Microsized Metallic Glasses, Scr. Mater, 2013, 68, p 773

Publisher's Note Springer Nature remains neutral with regard to jurisdictional claims in published maps and institutional affiliations. 